\title{
Obesity Markers as Predictors for Colorectal Neoplasia \\ (J Obes Metab Syndr 2017;26:28-35)
}

\author{
Jin-Hyun Kwon, Hae-Jin Ko*, Chang-Ho Youn, Hye-In Choi \\ Department of Family Medicine, Kyungpook National University School of Medicine, Daegu, Korea
}

*Corresponding author

Hae-Jin Ko

http://orcid.org/0000-0003-4460-1476

Department of Family Medicine, Kyungpook National University Hospital, 130 Dongduk-ro, Jung-gu, Daegu 41944, Korea

Tel: $+82-53-200-6578$

Fax: +82-53-200-5480

E-mail: liveforme@knu.ac.kr
Obesity is known to be an important risk factor that increases the prevalence of malignancies including colorectal cancer. ${ }^{1,2}$ Considering that colorectal cancers are mostly developed from adenomatous polyps through an adeno-carcinoma sequence, it is important to determine the association of colorectal neoplasia, including adenomatous polyps, with obesity in order to prevent colorectal cancer. However, there is a lack of studies investigating which obesity parameter would be the best index to predict colorectal neoplasia; hence, we studied the relationships among colorectal neoplasia, obesity, and obesity markers.

Our study revealed that obesity was significantly associated with colorectal neoplasia including tubular adenoma, advanced adenoma, or cancer; waist-to-height ratio was a better indicator of colorectal neoplasia than the other obesity markers. Among women, waist circumference was also a more useful marker than body mass index. This result might indicate that abdominal obesity is associated with an increased risk of colorectal cancer by the mechanisms of insulin resistance, hyperinsulinemia, chronic inflammation, and altered levels of growth factors. ${ }^{3}$

Our study adjusted for possible confounding variables that can influence the development of colorectal neoplasia, such as sex, smoking, drinking, and exercise. However, this study has a limitation in that we did not adjust for menopausal status in women, which is also an important variable related to colorectal neoplasia. Estrogen, especially estradiol, has revealed a protective effect against colorectal carcinogenesis through several mechanisms: reducing secondary bile acid production and circulating insulin like growth factor-1, stimulating humoral and cell-mediated immune responses, and inhibiting cell proliferation of colorectal tumors by binding to estrogen receptors like ER- $\beta{ }^{4-6}$ Menopausal status is related to increased risk of colorectal cancer, although menopausal hormone therapy might reduce the risk of colorectal cancer. ${ }^{7}$ In our study, the mean age of the female subjects was 46.27 years, and the majority (69.5\%) of the women were younger than 50 . Although we did not adjust for the use of hormone replacement, the influence of menopausal status was considered to be trivial.

Among the types of abdominal obesity, visceral adipose tissue (VAT) is plays a main role in hyperinsulinemia and preneoplastic colonic mucosal changes. ${ }^{8}$ Recently, new procedures to measure visceral adiposity have been developed. Among those, Nagata et al. ${ }^{9}$ revealed that VAT measured by computed tomography (CT) had a strong relationship with colorectal adenoma. Furthermore, a meta-analysis of the association of colorectal adenoma with VAT measured by abdominal CT showed that the amount of VAT was significantly associated with an increased risk of colorectal adenoma in both men and women. ${ }^{10}$ Since abdominal visceral obesity 
has a strong relation with colorectal neoplasia, the other tools to estimate VAT, such as dual-energy X-ray absorptiometry or magnetic resonance imaging, might have similar correlations with colorectal adenoma to CT.

Therefore, further studies using various imaging tools and anthropometric indices to confirm and predict abdominal obesity and prevent colorectal neoplasm are warranted. Medical care providers should give careful attention to obesity, especially abdominal obesity, and make greater efforts to control this condition.

\section{CONFLICTS OF INTEREST}

The authors declare no conflict of interest.

\section{REFERENCES}

1. Koh SJ, Kim JS. The reasons for the increased incidence of colorectal cancer in Korea. Korean J Med 2010;79:97-103.

2. Morita T, Tabata S, Mineshita M, Mizoue T, Moore MA, Kono $\mathrm{S}$. The metabolic syndrome is associated with increased risk of colorectal adenoma development: the Self-Defense Forces health study. Asian Pac J Cancer Prev 2005;6:485-9.

3. Jochem C, Leitzmann M. Obesity and colorectal cancer. Recent Results Cancer Res 2016;208:17-41.
4. Newcomb PA, Pocobelli G, Chia V. Why hormones protect against large bowel cancer: old ideas, new evidence. Adv Exp Med Biol 2008;617:259-69.

5. Hsu HH, Kuo WW, Ju DT, Yeh YL, Tu CC, Tsai YL, et al. Estradiol agonists inhibit human LoVo colorectal-cancer cell proliferation and migration through p53. World J Gastroenterol 2014;20:16665-73.

6. Kennelly R, Kavanagh DO, Hogan AM, Winter DC. Oestrogen and the colon: potential mechanisms for cancer prevention. Lancet Oncol 2008;9:385-91.

7. Park SY, Wilkens LR, Kolonel LN, Henderson BE, Le Marchand L. Inverse associations of dietary fiber and menopausal hormone therapy with colorectal cancer risk in the Multiethnic Cohort Study. Int J Cancer 2016;139:1241-50.

8. Frezza EE, Wachtel MS, Chiriva-Internati M. Influence of obesity on the risk of developing colon cancer. Gut 2006;55:28591.

9. Nagata N, Sakamoto K, Arai T, Niikura R, Shimbo T, Shinozaki M, et al. Visceral abdominal fat measured by computed tomography is associated with an increased risk of colorectal adenoma. Int J Cancer 2014;135:2273-81.

10. Hu H, Cai Y, Huang J, Zhang J, Deng Y. Visceral adipose tissue and the risk of colorectal adenomas: a meta-analysis of observational studies. Eur J Cancer Prev 2015;24:462-9. 